\title{
ST Elevation in a Professional Athlete: From the Coronary Care Unit to the Field
}

\author{
Maria Boutsikou ${ }^{1,2}$, Elias Sanidas ${ }^{3}$, Gkiouzelis Giasemis $^{4}$, George Ziogas ${ }^{5}$, George Dangas ${ }^{6}$, \\ Panagiotis Stamatopoulos ${ }^{1, *}$
}

${ }^{1}$ Department of Pediatric, Athens University Medical School, Children's Hospital “Aghia Sophia”, Athens, Greece

${ }^{2}$ Mediterraneo Hospital, Athens, Greece

${ }^{3}$ Department of Cardiology, Laiko General Hospital, Athens, Greece

${ }^{4}$ Agios Paulos General Hospital, Thessaloniki, Greece

${ }^{5}$ Sportsclinic, Thessaloniki, Greece

${ }^{6}$ Icahn School of Medicine at Mount Sinai, New York, USA

Email address:

giropanstam@gmail.com (P. Stamatopoulos)

${ }^{*}$ Corresponding author

\section{To cite this article:}

Maria Boutsikou, Elias Sanidas, Gkiouzelis Giasemis, George Ziogas, George Dangas, Panagiotis Stamatopoulos. ST Elevation in a Professional Athlete: From the Coronary Care Unit to the Field. Cardiology and Cardiovascular Research. Vol. 3, No. 4, 2019 , pp. 86-89. doi: $10.11648 /$ j.ccr.20190304.12

Received: July 10, 2019; Accepted: September 17, 2019; Published: September 30, 2019

\begin{abstract}
Interpretation of ECG in athletes can be quite challenging as it requires adequate experience to distinguish physiologic changes related to adaptation to training from pathologic findings. ECG interpretation is an important diagnostic tool for high intensity trained athletes. Herein, we present the case of a young high-competitive athlete who presented with a variant ST elevation on 12lead ECG that was initially considered suspicious of myocardial infarction. Diagnostic workup including clinical examination, echocardiogram, 12-lead surface ECG and laboratory tests (troponin and CK-MB) did not confirm the presence of acute coronary syndrome. The patient was monitored for a few hours and was finally discharged with no restrictions regarding training. ECG in the present case resembles Wellen's syndrome pattern which represents a preinfarction stage of CAD with a significant proximal left anterior descending artery (LAD) stenosis that will be followed by ACS of anterior wall if left untreated. However, there were no other findings to support the clinical diagnosis of Wellen's syndrome in our case and the ECG changes were attributed to a physiological adaptation to training. During exercise several ECG adjustments develop as a result of the decreased sympathetic tone and the increased parasympathetic tone combined with the heterogeneity of ventricle repolarization. The preparticipation screening performed by experienced physicians in the field of sports cardiology is crucial in order to recognize physiologic cardiovascular adaptation to exercise and exclude cardiovascular abnormalities.
\end{abstract}

Keywords: ECG, ST Elevation, Biphasic T Waves, Early Repolarization, Athlete, Training, Wellen's Syndrome

\section{Introduction}

Electrocardiogram (ECG) is an important diagnostic tool for high intensity trained athletes. Nonetheless, the ECG interpretation might be quite challenging as it requires adequate experience to distinguish physiological changes related to cardiac adaptation to training from pathological findings [1]. Diagnostic evaluation should always include a careful personal and family history of heart disease or sudden cardiac death along with a detailed clinical examination and ECG. ST segment elevation on ECG that is a common finding in acute coronary syndromes (ACS) could generate concern in a professional athlete. However, this feature is not always related to myocardial ischemia. Herein, we present a case of a young high-competitive black basketball player that was presented with a variant ST segment elevation which was initially considered suspicious of ACS. 


\section{Case Information}

A 27-year old black male professional basketball player with no previous medical history of coronary artery disease (CAD) underwent clinical screening as part of his regular medical check-up. The patient also denied any family history of CAD, cardiomyopathy or sudden cardiac death. He was completely asymptomatic. His blood pressure (BP) was within normal limits and heart auscultation revealed normal heart sounds with no additional murmurs while his blood work was unremarkable. Nevertheless, his ECG was considered abnormal, suggestive of ACS. Thus, he was referred to the Cardiology Care Unit where further clinical examination, ECG monitoring along with laboratory and imaging tests were performed.

In particular, ECG showed sinus rhythm (heart rate of 57bpm), normal QRS axis and normal PR and QTc intervals (160 msec and $390 \mathrm{msec}$, respectively). However, a notable upward convex ST segment elevation ( $\max 3 \mathrm{~mm}$ ) with concave biphasic $\mathrm{T}$ waves in V2-V3 leads were present. In addition, there was a notching on the downstroke of $\mathrm{R}$ waves in V4-V6 leads, consistent with J point elevation (Figure 1).

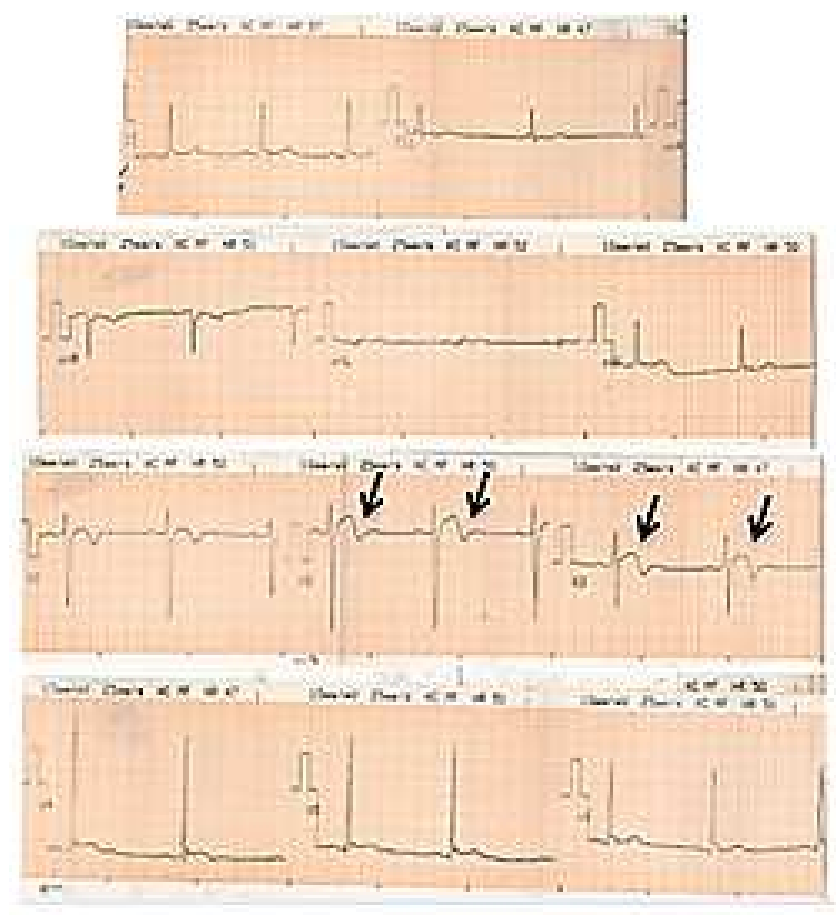

Figure 1. Patient's ECG on admission.

The patient was scheduled for transthoracic echocardiography (TTE) that revealed normal biventricular size with normal left ventricular (LV) ejection fraction $(\mathrm{EF}=$ $60 \%$ ) and no regional wall motion abnormalities. No LV hypertrophy (LVH) or right ventricular hypertrophy (RVH) was present. There was prolapse of the anterior and posterior mitral valve leaflets with mild mitral regurgitation. Mild tricuspid regurgitation was also observed. The aortic root along with the mitral and pulmonary valves were normal. There were no other cardiac defects or signs of rheumatic valve disease. Troponin and creatine kinase-MB (CK-MB) plasma levels were within normal limits. Thereinafter, a stress echocardiography was performed that was negative for inducible ischemia on maximum exercise since he succeeded $85 \%$ of predicted for his age with normal BP response.

\section{Result}

The patient was monitored for a few hours and was finally discharged after there were no ECG changes or troponin rise on serial testing. No restriction in regards to his exercise activity was applied at that point. Unfortunately, the patient moved abroad and, thus, no further information on his follow up is available.

\section{Discussion}

We presented a case of an interesting variant of ST segment elevation in a high-competitive professional black basketball player, presumably suggestive of an early repolarization that was initially considered suspicious of severe ACS. In particular, a notable upward convex ST segment elevation with biphasic $\mathrm{T}$ waves was noted in V2V3 leads on surface ECG as part of his regular medical check-up.

High intensity training is responsible for electrical and structural cardiac adaptations that are referred with the term "athlete's heart". During exercise the decreased sympathetic tone and the increased parasympathetic tone combined with the heterogeneity of ventricle repolarization promotes several ECG adjustments. Such changes are commonly considered as benign variants and represent a physiological adaptation to chronic training. They do not need further investigation in the absence of symptoms and normal physical examination. Nevertheless, distinguishing them from findings indicating cardiac pathology might be quite difficult $[2,3]$.

The most common training related ECG alterations are rhythm disturbances and conduction abnormalities (sinus bradycardia, ectopic atrial rhythm, $1^{\text {st }}$ degree atrioventricular (AV) block, $2^{\text {nd }}$ degree AV block Mobitz type I, incomplete right bundle branch block - RBBB) as well as early repolarization. The last one is described as convex ST segment elevation combined with inverted $\mathrm{T}$ waves in $\mathrm{V} 1-\mathrm{V} 4$ leads mainly observed in black / African athletes [4, 5].

In a recent study of 519 National Basketball Association athletes' ECGs, early repolarization referred as convex ST segment elevation and $\mathrm{T}$ wave inversion in V2-V4 leads was the most frequent finding in black / African players. In the same study it was mentioned that abnormal findings were more prevalent in older athletes suggesting a possible correlation between long term high intensity training and ECG abnormalities [6]. In our case, ECG showed sinus bradycardia and ST segment changes that were in consistence with the above data. However, the presence of biphasic $\mathrm{T}$ waves in V2-V3 leads was not typical. Given the fact that detailed cardiac evaluation did not revealed cardiac pathology, this feature might potentially be an interesting ECG variation of early repolarization among high- 
competitive black athletes confirming the concept of athlete's heart.

Other ECG features that do not need further investigation when they are observed in isolation in a high-competitive athlete include [1] left or right atrial enlargement, [2] left or right axis deviation and [3] complete RBBB. Nevertheless, the presence of two or more of the above findings is considered abnormal and further cardiac evaluation is mandatory. Conversely, training unrelated ECG features are ST segment depression, pathological Q waves, left bundle branch block (LBBB), atrial or ventricular arrhythmias, $\mathrm{T}$ wave inversion beyond V1 in white athletes or V4 in black athletes, Brugada ECG pattern, QTc $\geq 470 \mathrm{msec}$ in men and $\geq 480 \mathrm{msec}$ in women and two or more premature ventricular contractions (PVCs) per 10 seconds. Although sinus bradycardia is a normal ECG finding in high intensity trained athletes, profound sinus bradycardia $(<30 \mathrm{bpm})$ requires further diagnostic cardiac investigation. PR interval $\geq 400 \mathrm{msec}, 2^{\text {nd }}$ degree AV block Mobitz II and $3^{\text {rd }}$ degree AV block are also abnormal findings that are not correlated with regular training and secondary evaluation will guide clinical decisions [2]. Fortunately, none of the above findings was present on our patient's surface ECG.

ECG in the present case resembles Wellen's syndrome pattern. Wellen's syndrome, primarily described by de Zwann et al. [7], represents a pre-infarction stage of CAD with a significant proximal left anterior descending artery (LAD) stenosis that will be followed by ACS of anterior wall if left untreated. There are two types: type A that accounts for almost $75 \%$ of all cases and is characterized by deeply inverted $\mathrm{T}$ waves in V2-V3 leads and type B that occurs in the rest of the cases and biphasic $\mathrm{T}$ waves are found in V2V3 leads (Figure 2). Neither Q waves nor significant ST segment elevation are observed. These ECG features are prevalent in pain free periods in a patient with a history of angina. Cardiac markers are normal or slightly elevated. During chest pain, upright $\mathrm{T}$ waves with possible ST segment elevation or depression are detected [8,9]. Our patient presented with biphasic $\mathrm{T}$ waves in V2-V3 leads. However, there were no other findings to support the clinical diagnosis of Wellen's syndrome. The athlete was asymptomatic and there were no ECG changes or biomarkers' levels elevation during his hospitalization. In addition, although the presence of biphasic $\mathrm{T}$ waves could be potentially suspicious, the convex elevation of ST segment was not concordant with Wellen's syndrome. ECG differences between our patient and Whellen's syndrome type B are illustrated by arrows in the Figure.

Differential diagnosis of our patient's ECG might also include myocarditis, pericarditis, Brugada syndrome and arrhythmogenic right ventricular cardiomyopathy (ARVC). Nonetheless, there were no chest pain, cardiac enzymes elevation and wall motion abnormalities. Furthermore, the different ECG features in each one of these conditions imply that in our case ECG represents a variant of early repolarization as part of athlete's heart concept.

The preparticipation screening is imperative in order to detect potentially lethal cardiovascular abnormalities. It should be performed by a physician with the specific education and medical skills to recognize clinical symptoms and signs related to cardiovascular diseases with risk for sudden cardiac death. It is of particular interest the existence of disagreement between European and American guidelines concerning the role of ECG in the preparticipation screening and, specifically, whether the addition of ECG to athletic cardiac evaluation is superior to history and physical examination alone for detecting cardiac pathology. European Society of Cardiology (ESC) recommends the performance of ECG in all athletes while American Heart Association (AHA) does not mention it as a routine screening tool $[2,4,10]$.

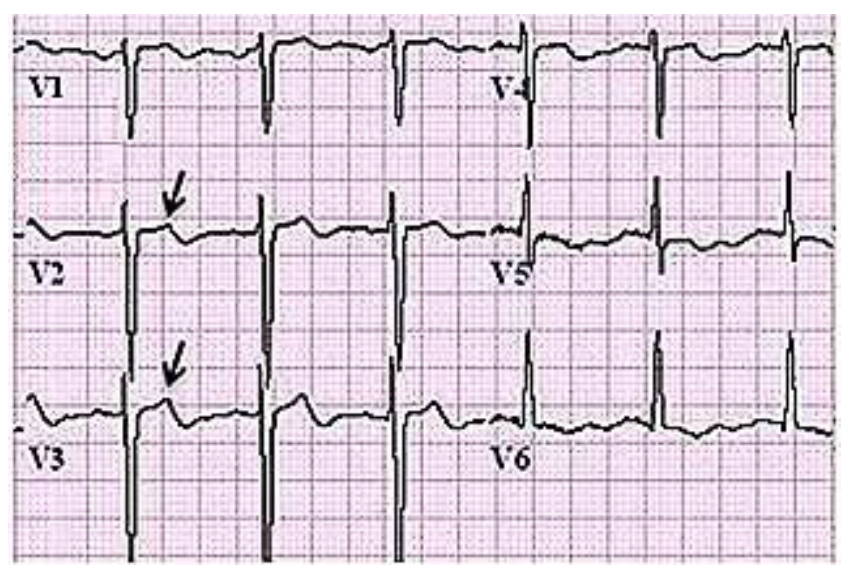

Figure 2. Whellen's syndrome type B ECG pattern. Concave biphasic $T$ waves are mentioned by arrows. Convex ST elevation, that is illustrated in our patient, does not exist in Whellen's syndrome type $B$.

Last but not least, there are concerns regarding the addition of echocardiography in the basic preparticipation screening. Echocardiography is a relatively inexpensive technique and the incorporation of this imaging modality into existing protocol could optimize the cardiac evaluation identifying athletes that require further investigation and detecting potentially lethal cardiovascular conditions $[11,12]$.

\section{Conclusion}

In conclusion, ST segment elevation is a common ECG finding suggestive of ACS. However, it might be also observed in healthy athletes mainly of black race as part of physiological cardiac adaptation to training.

\section{Recommendations}

In such cases, a thorough detailed personal and family history as well as clinical examination and ECG could safely guide diagnosis. These patients should be advised to undergo at least one advanced imaging modality and have a copy of their ECG with them. Finally, they should be aware of suspicious symptoms or signs including chest pain, palpitation and dyspnea that require immediate medical care. 


\section{Author Contributions}

M. Boutsikou, I. Sanidas and G. Giasemis are responsible for writing the case report. P. Samatopoulos and G. Ziogas clinically assessed the patient and contributed to the manuscript editing. G Ntangas reviewed and edited the final version of the manuscript.

\section{Conflict of Interest Statement}

There is no conflict of interest for this case report.

\section{Acknowledgements}

No.

\section{References}

[1] Van der Wall EE. ECG screening in athletes: optional or mandatory? Neth Heart J 2015; 23: 353-5.

[2] Sharma S, Drezner JA, Baggish A et al. International Recommendations for Electrocardiographic Interpretation in Athletes. J Am Coll Cardiol 2017; 69: 1057-1075.

[3] Pluim BM, Zwinderman AH, van der Laarse A, van der Wall EE. The athlete's heart. A meta-analysis of cardiac structure and function. Circulation 2000; 101: 336-44.

[4] Sheikh N, Papadakis M, Ghani S et al. Comparison of electrocardiographic criteria for the detection of cardiac abnormalities in elite black and white athletes. Circulation 2014; 129: 1637-49.

[5] Sharma S, Merghani A, Mont L. Exercise and the heart: the good, the bad, and the ugly. Eur Heart J 2015; 36: 1445-53.

[6] Waase MP, Mutharasan RK, Whang W et al. Electrocardiographic Findings in National Basketball Association Athletes. JAMA Cardiol 2018; 3: 69-74.

[7] de Zwaan C, Bar FW, Wellens HJ. Characteristic electrocardiographic pattern indicating a critical stenosis high in left anterior descending coronary artery in patients admitted because of impending myocardial infarction. Am Heart J 1982; 103: 730-6.

[8] Miner B, Hart EH. Wellens Syndrome. StatPearls. Treasure Island (FL), 2018.

[9] Agarwal A, Vyas S, Kumar R. Wellen's syndrome: Challenges in diagnosis. Malays Fam Physician 2015; 10: 35-7.

[10] Drezner JA, Ackerman MJ, Anderson J et al. Electrocardiographic interpretation in athletes: the 'Seattle criteria'. Br J Sports Med 2013; 47: 122-4.

[11] Grazioli G, Merino B, Montserrat S et al. Usefulness of echocardiography in preparticipation screening of competitive athletes. Rev Esp Cardiol (Engl Ed) 2014; 67: 701-5.

[12] Mitchell AR, Hurry R, Le Page P, MacLachlan H. Preparticipation cardiovascular screening: is community screening using hand-held cardiac ultrasound feasible? Echo Res Pract 2015; 2: 49-55. 